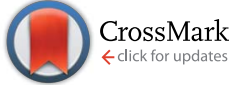

Cite this: RSC Adv., 2017, 7, 7303

Received 1st December 2016 Accepted 9th January 2017

DOI: 10.1039/c6ra27609a

www.rsc.org/advances

\section{Polymerized high internal phase emulsion monolithic material: a novel stationary phase of thin layer chromatography}

\begin{abstract}
Dezhong Yin, ${ }^{\star a}$ Yudong Guan, ${ }^{a}$ Huimin Gu, ${ }^{b}$ Yu Jia ${ }^{a}$ and Qiuyu zhang ${ }^{\star b}$
A polymerized high internal phase emulsion (polyHIPE) was prepared using styrene, divinylbenzene and butyl acrylate as raw material and employed as stationary phase of thin layer chromatography (TLC). SEM, mercury intrusion porosimetry and FT-IR spectrum were employed to characterize the pore structure and chemical composition. To verify the feasibility as stationary phase of TLC, identification of plant extracts, Chinese herbs and traditional Chinese medicine were practiced on the prepared polyHIPE. Relative retardation factor $(\alpha)$, theoretical plate number $\left(N_{\text {plate }}\right)$ and resolution $(R)$ were used to evaluate the performance of TLC. Large difference in $\alpha$ value make it is feasible to optimize the chromatographic condition. The resolution $R$ and $N_{\text {plate }}$ indicates that the introduction of BA in polyHIPE facilitates the preparation and improves the performance for TLC separation. Our results show that polyHIPE monolith is a reusable stationary phase of TLC with high performance.
\end{abstract}

\section{Introduction}

Thin-layer chromatography (TLC), with a thin layer sorbent as separation medium, is mostly used to monitor the progress of organic reactions, to identify compounds in a given mixture, and to determine product purity. The main advantages of TLC are the use of inexpensive equipment, lower purity of consuming solvents, saving a significant amount of consumables..$^{1-3}$ It is important to select a suitable stationary phase when using TLC to separate mixtures. Thin chromatographic layers can be formed from different organic and inorganic sorbents ${ }^{4,5}$ and different plates are commercially available. The most commonly used conventional TLC plates consist of sorbent layers formed from irregular $c a .10 \mathrm{~mm}$ silica particles held together by a binder.

Recent year, several new stationary phases ${ }^{6-8}$ have been developed, providing users alternative for more difficult chromatographic separations. Hollow silica microspheres with different structures have been synthesized and used to separate a model mixture of methyl red and dimethyl yellow. ${ }^{9}$ A comparison test shows that HSMs as a stationary phase are advantageous over commercial silica gel in an easy preparation of homogeneous TLC thin plates. Another kind of silica gel, gold nanoparticles grafted 3-triethoxysilyl propylamine modified silica gel was developed as a stationary phase for

${ }^{a}$ School of Science, Northwestern Polytechnical University, Xi'an 710072, China. E-mail: dezh_yin@mwpu.edu.cn

${ }^{b}$ Key Laboratory of Space Applied Physics and Chemistry, Ministry of Education, Northwestern Polytechnical University, Xi'an, Shaanxi, 710072, China. E-mail: qyzhang1803@gmail.com separation and determination of two steroid hormones, namely progesterone and testosterone. ${ }^{10}$ Validity of the method was investigated, and a wide linear range of 1-200 ng per spot, and low detection limits of $0.16 \mathrm{ng}$ and $0.13 \mathrm{ng}$ per spot, low quantification limits of $0.51 \mathrm{ng}$ and $0.40 \mathrm{ng}$ per spot, and good precision (expressed as percent relative standard deviation) lower than $3.1 \%$ and $2.7 \%$ were obtained for progesterone and testosterone. A DNA/PVA interpenetrating polymer network was used to coat the surface of the porous silica particles for TLC. ${ }^{11}$

Monolith, a single continuous rod of highly porous material with an interconnected network of pores, is demonstrated to be an excellent candidate as ideal stationary phase of chromatography. ${ }^{12,13}$ For TLC analysis, several monoliths have been successfully demonstrated. A poly(butylmethacrylate-coethylene dimethacrylate) layer was prepared and used for separation of peptides and proteins, followed by MALDI-TOFMS detection. ${ }^{14}$ Two-dimensional thin layer chromatography of peptides was also carried out on a superhydrophobic monolithic porous polymer layers coupled with desorption electrospray ionization mass spectrometer ${ }^{15}$ or matrix-assisted laser desorption/ionization (MALDI) mass spectrometry. ${ }^{16} \mathrm{~F}$. Svec prepared porous hypercrosslinking styrenics-based monolithic layers via a Friedel-Crafts alkylation reaction then formed a multiplicity of mesopores that increased the surface area and enabled separation of small dye molecules. ${ }^{17}$ Polyamide was also used in TLC to separate catechins and Omethylated (-)-epigallocatechin gallate. ${ }^{18}$

High internal phase emulsions (HIPEs), an emulsion with internal phase exceeds $74 \%$, has been found considerable to create highly porous polymer monoliths (referred as 
polyHIPE). ${ }^{19,20}$ By large amount of surfactants, ${ }^{21}$ a large portion of internal phase can be stabilised in the continuous phase to form stable HIPE. After the polymerisation, polyHIPE materials with a typical inter-connected porous morphology, consisting of large primary pores(cavities) and smaller interconnecting pores, are obtained. Due to special properties of low density, high porosity, interconnected pore structure and high permeability, ${ }^{22}$ polyHIPE is used in as adsorbents, ${ }^{23}$ catalyst carriers, ${ }^{24}$ separation medium. ${ }^{25}$

It raised significant interest in the polyHIPE as stationary phase of TLC due to the well-defined monolithic shape and interconnected pore structure of polyHIPE. In this paper, porous polyHIPE monolithic plates were prepared and used as stationary phase of TLC for identification of Chinese herbal medicinal ingredients. To the best of our knowledge, this is the first demonstration of polyHIPE as stationary phase of TLC.

\section{Experimental}

\subsection{Chemicals}

Styrene (St, $99 \mathrm{wt} \%$ ), divinylbenzene (DVB, $80 \mathrm{wt} \%$ ), benzoyl peroxide (BPO, 99 wt\%) were purchased from Sinopharm Chemical Reagent Co. (Shanghai, China). Butyl acrylate (BA), $\mathrm{CaCl}_{2}$ and sorbitan monooleate (span80) were purchased from Fucheng Chemical Reagent Co. (Tianjing, China). Three herb extracts (emodin, chrysophanol, tanshinone), two herbs (Rhizoma Chuanxiong and Polygonum Cuspidatum) were purchased from National Institute for food and drug Control of China. All the chemicals were of analytical grade and used as received.

\subsection{Preparation of polyHIPE plate}

A water-in-oil HIPE was prepared by mixing oil phase and aqueous phase (1.0 wt\% $\mathrm{CaCl}_{2}$ solution) and emulsifying by homogenizer, as reported elsewhere. ${ }^{26,27}$ As a typical procedure, monomer (including St, BA, DVB), BPO and span 80 were mixed to form oil phase. $\mathrm{CaCl}_{2}$ aqueous solution (1.0 wt\%) was added dropwise under constant stirring at $3000 \mathrm{rpm}$. Once all aqueous phase has been added, stirring was continued for a further 5 min to produce a uniform milky W/O HIPE. To assemble a rectangle mould for curing the emulsion, four slides (thickness $2 \mathrm{~mm}$ ) was put on a glass plate (Fig. 1a).
Then viscous HIPE was poured into the mold (Fig. 1b) and covered with a glass plate to prevent the evaporation of monomer during following curing process (Fig. 1c). After the polymerization was completed for 8 hours in $70^{\circ} \mathrm{C}$ water-bath, the mould was disassembled and a monolithic plate was obtained (Fig. 1d). Finally, the plate was washed in ethanol ultrasonically to remove the impurities and a polyHIPE plate of $2 \mathrm{~mm}$ thickness was obtained.

Optical micrographs were collected with an optical microscope equipped with a digital camera (CAIKON, China). FTIR spectrum (Fourier transformation infrared) was obtained using a BRUKER Tensor 27 spectrometer. Morphology was characterized by a JSM-6700 F Scanning electron microscope (Japan) equipped with a field emission electron gun. The total pore area of polyHIPE was characterized by mercury porosimetry (AUTOPORE IV 9500, Micromeritics, USA). The porosity of polyHIPE was measured using pyncometry. The foam density $\left(\rho_{\mathrm{f}}\right)$ of polyHIPEs was measured on approximately 3 $\mathrm{cm}^{3}$ of broken pieces of each sample. The matrix density $\left(\rho_{\mathrm{m}}\right)$ was determined using a pycnometer using approximately $500 \mathrm{mg}$ of crushed polyHIPEs and ethanol as medium. The porosity $(P)$ of polyHIPE was calculated according to the following equation: ${ }^{28}$

$$
P=\left(1-\frac{\rho_{\mathrm{f}}}{\rho_{\mathrm{m}}}\right) \times 100 \%
$$

\subsection{TLC analysis}

For the TLC analysis, solutions preparation and TLC separation were practiced according to the Chinese pharmacopoeia. Herb extracts were dissolved in ethanol $\left(1 \mathrm{mg} \mathrm{mL}^{-1}\right)$. For TLC of herbs, $1.0 \mathrm{~g}$ herb or herbs mixture were extracted ultrasonically for 15 minutes in methanol. Then the solution was separated by filter and concentrated to $2 \mathrm{~mL}$. A second filter was practised to get a clean solution for TLC. $5 \mu \mathrm{L}$ of solution was spotted at one end of polyHIPEs plate by using a glass capillary and developed in a TLC tank containing mobile phase. The time and the distance of TLC develop was recorded and the permeability of monolith was calculated by dividing the distance with the time of TLC develop. After development, the plate was air-dried and visualized under daylight or ultraviolet (365 nm). To make the monolith plate reusable, polyHIPE was washed by methanol ultrasonically. Relative retardation factor $(\alpha)$, theoretical plate

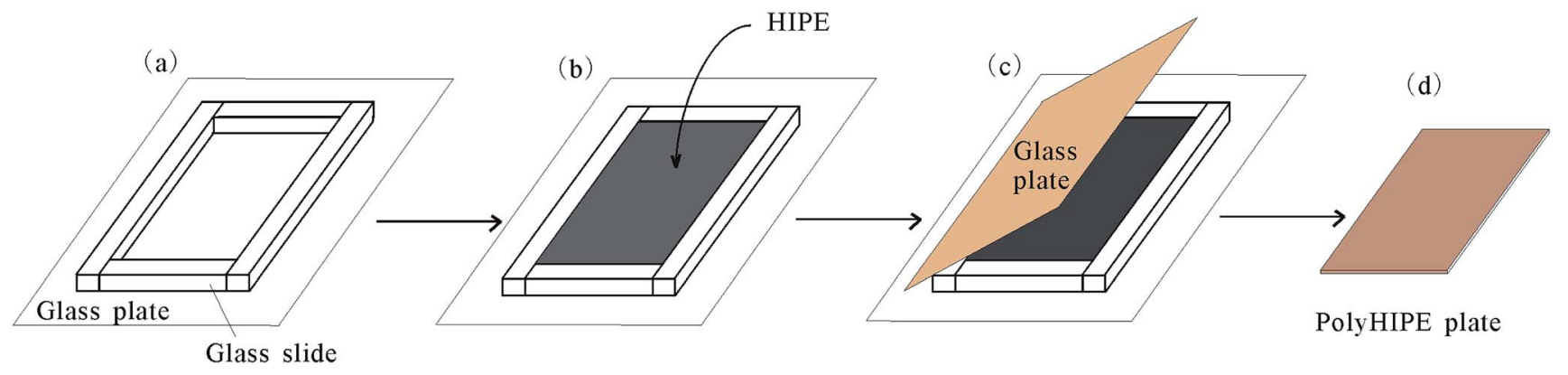

Fig. 1 Schematic illustration of preparation of polyHIPE plate. 
number $\left(N_{\text {plate }}\right)$ and resolution $(R)$ were used to evaluate the performance of TLC, according to equations:

$$
\begin{gathered}
\alpha_{2,1}=\frac{R_{\mathrm{f}, 2}}{R_{\mathrm{f}, 1}} \\
N_{\text {plate }}=16\left(\frac{L}{W-W_{0}}\right)^{2} \\
R=\frac{2\left(L_{2}-L_{1}\right)}{W_{2}+W_{1}}
\end{gathered}
$$

(2) In this study, we applied a process developed previously in our

where $R_{\mathrm{f}}$ is the rate of flow of an analyte, $L$ is the distance travelled by analyte, $W_{0}$ and $W$ are the spot width before and after development.
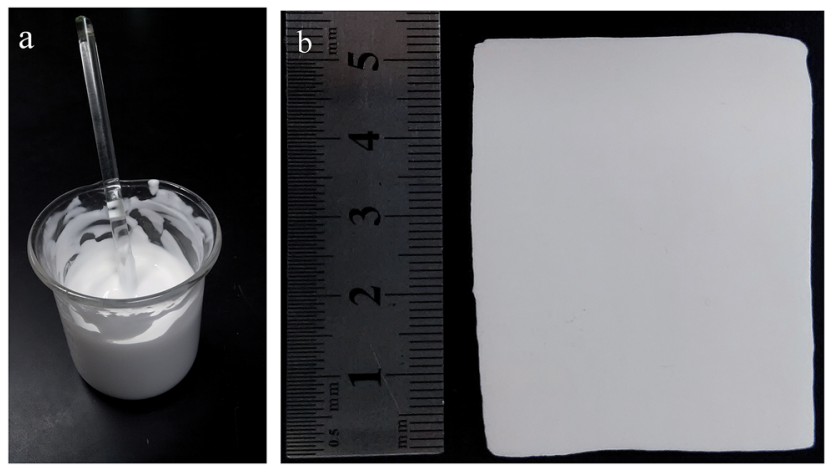
$\mathrm{lab}^{\mathbf{6}, 29}$ for the preparation of HIPE. Span 80 was developed as the most efficient stabilizer for styrenics-based HIPE with high

\section{Results and discussion}

\subsection{Preparation of HIPE and polyHIPE}

viscosity. We employed St $\sim$ DVD to prepare polyHIPE under different Span 80 amount. Emulsion under 5\% of Span 80 phase separated because of the Ostwald ripening which result in large droplets. Under $10 \%$ and $20 \%$ of Span 80 , the emulsion is stable for a long time of more than 1 week (Fig. 2a).

A typical advantage of polyHIPE is that it can replicate the shape of the mould. As shown schematically in Fig. 1, we assembled a mould by putting four glass slides on a large glass plate. HIPE is ice cream-like and can be hold in the mould even the mould is not well sealed. By covered with another glass plate, the HIPE was confined in the rectangle mould and the thickness of glass slide defined the thickness of polyHIPE. After polymerization, the PSt polyHIPE monolith adhered to the bottle plate. Upon disassembling the mould, carefulness is necessary because the PSt polyHIPE is brittle, fragile and easy to be broken. To improve this, a soft monomer BA is added as comonomer. The obtained $\mathrm{P}(\mathrm{St}-\mathrm{Co}-\mathrm{BA})$ plate is much more flexible and easy to be de-patched away from the glass slide to be a smooth and regular plate (Fig. 2b). Four polyHIPE plates with different amount of Span80 and BA monomer were obtained, as listed in Table 1.

\begin{tabular}{|c|c|c|c|c|c|c|c|c|}
\hline PolyHIPE $^{a}$ & St $(g)$ & DVB (g) & BA $(g)$ & Span80 $(\mathrm{g})$ & $\begin{array}{l}\mathrm{CaCl}_{2} \text { solution } \\
(\mathrm{mL})\end{array}$ & BPO (mg) & $\begin{array}{l}\text { Porosity } \\
(\%)\end{array}$ & $\begin{array}{l}\text { Total pore area } \\
\left(\mathrm{m}^{2} \mathrm{~g}^{-1}\right)\end{array}$ \\
\hline PS10 & 4 & 1 & - & 0.5 & 15 & 50 & 65.7 & 25.5 \\
\hline PS10BA20 & 3.2 & 0.8 & 1.0 & 0.5 & 15 & 50 & 74.9 & 25.3 \\
\hline PS20BA20 & 3.2 & 0.8 & 1.0 & 1.0 & 15 & 50 & 71.0 & 23.8 \\
\hline
\end{tabular}

Fig. 2 Typical HIPE (a) and polyHIPE (b).

Table 1 Recipe for four different plates and pore characterization

${ }^{a} \mathrm{P}$ refers to polyHIPE. The number after S and BA refers to the percentage of span 80 and butyl acrylate (respective to monomer), respectively.
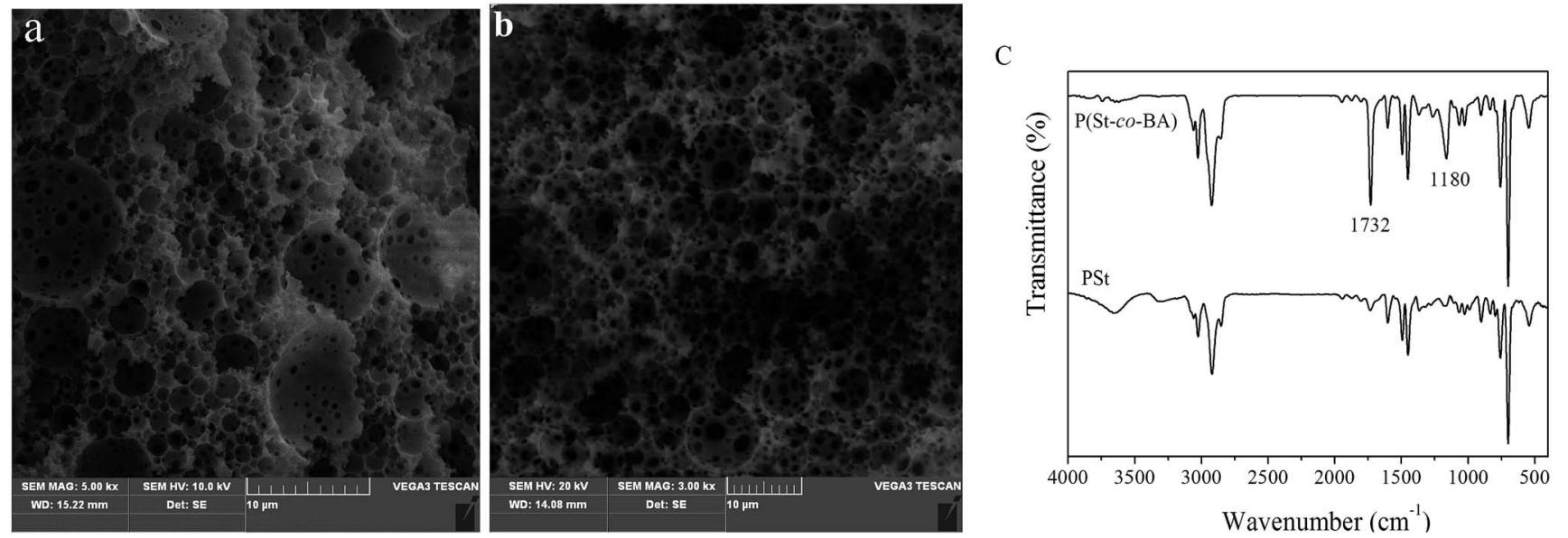

Fig. 3 SEM of PSt plate (a), P(St-Co-BA) plate (b) and FTIR spectra of PSt and P(St-co-BA) plate (c). 


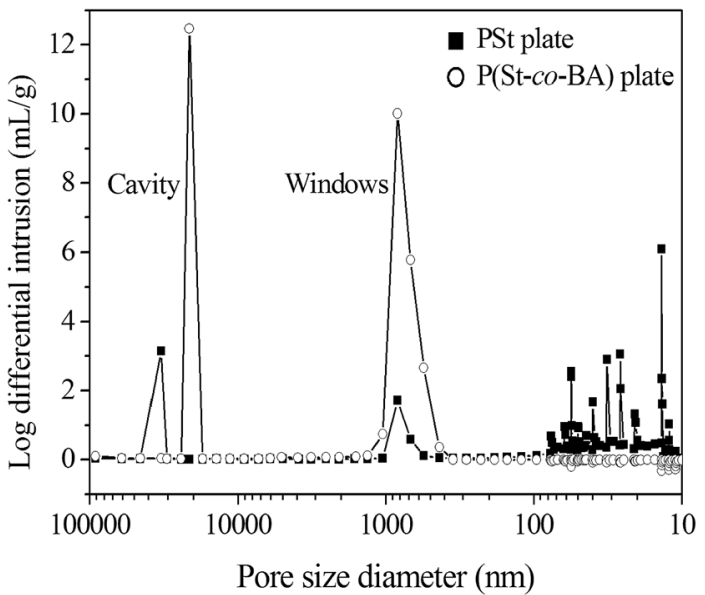

Fig. 4 Pore size distribution of PSt plate and P(St-co-BA) plate.

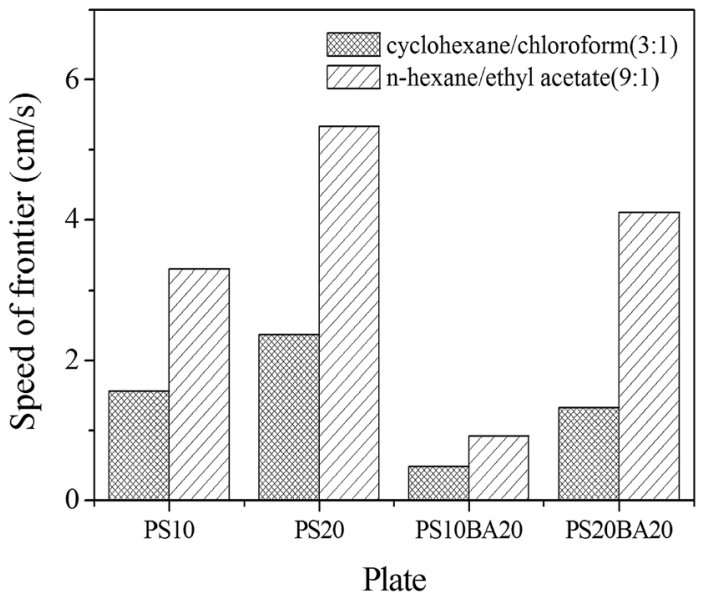

Fig. 5 Speed of frontier of mobile phase on different plate.

\subsection{Characterization of polyHIPE}

The inner structure of prepared polyHIPE was examined by SEM. By polymerized the monomer in the continuous phase of HIPE, a polyHIPE monolith can be formed to be a replica of HIPE. After washed with ethanol to remove the surfactant, typical open-cellar porous polyHIPE monoliths, both the inner and the surface of polyHIPE, were obtained. This pore structure was consistent with the result reported by Svec. ${ }^{17}$ The SEM images show typical primary cavities $(5-20 \mu \mathrm{m})$ and interconnecting windows $(0.5-1 \mu \mathrm{m})$ on the wall (Fig. 3a and b). FT-IR spectrum of $\mathrm{P}(\mathrm{St}-c o-\mathrm{BA})$ plate presents the characteristic peaks of styrene $\left(1600,1448\right.$ and $\left.698 \mathrm{~cm}^{-1}\right)$ and butyl acrylate $\left(1730 \mathrm{~cm}^{-1}\right.$ by carbonyl vibration) (Fig. 3c). This result shows that styrene and butyl acrylate was copolymerized in the plate.

Porosity and total pore area was employed to evaluate the pore property. The porosity ranges from $71-75 \%$, consistent with the theoretical value of $74 \%$. The porosity of PS10 decreased to be only $66 \%$, probably caused by the destabilization of HIPE during polymerization..$^{29}$ Mercury intrusion porosimetry was employed to evaluate the total pore area. As shown in Fig. 4, two groups of pore in micrometer size were observed. The pore of $0.4-1.0 \mu \mathrm{m}$ was originated from the windows on the wall, while the pores of tens of micros represent the cavity of polyHIPE. This result was somewhat different from other's report where they claim that the size of windows can be detected because of the distinctive mechanism of mercury intrusion porosimetry. ${ }^{30}$ The size of windows was typically range from $0.4-1.0 \mu \mathrm{m}$, irrespective of the employment of monomer BA. However, the size of cavities in $\mathrm{P}(\mathrm{St}-c o-\mathrm{BA})$ plate is much smaller than that in PSt plate, resultantly, $\mathrm{P}(\mathrm{St}-\mathrm{co}-\mathrm{BA})$ plate is much more homogeneous than PSt plate. Because of the improvement of homogeneity in cavities, the flow channel of mobile phase in $\mathrm{P}$ (St-co-BA) plate is much more homogeneous than that in PSt plate,
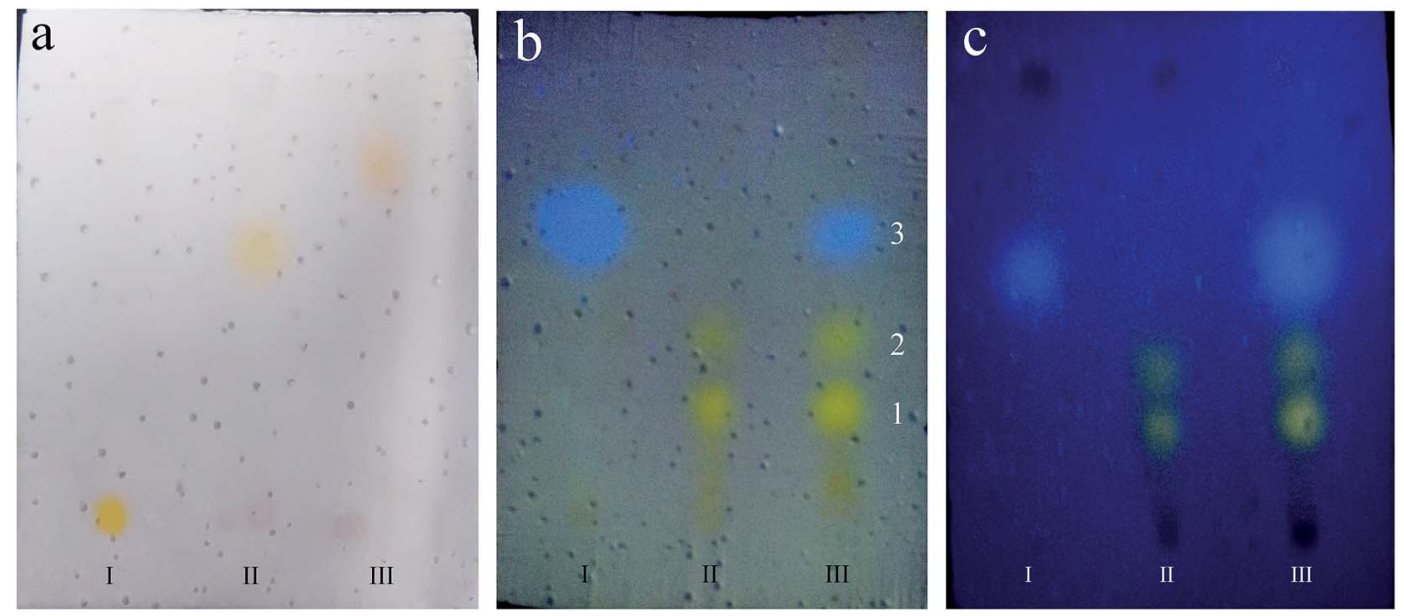

Fig. 6 TLC images of (a) herb extracts, (b) herbs on P(St-co-BA) plate (after reused five times) and (c) herbs on P(St-co-BA) plate (after reused 10 times). (a) I - emodin, II - chrysophanol, III - tanshinone. Developed in cyclohexane/chloroform (3 : 1) and viewed under daylight. (b, c) I Chuanxiong, II - Cuspidatum, III - mixture of Chuanxiong, Cuspidatum and Scutellaria. Developed in $n$-hexane/ethyl (9:1) and viewed under UV $365 \mathrm{~nm}$. 
Table 2 Separation efficiency to herb extracts and herbs on P(St-co-BA) plates

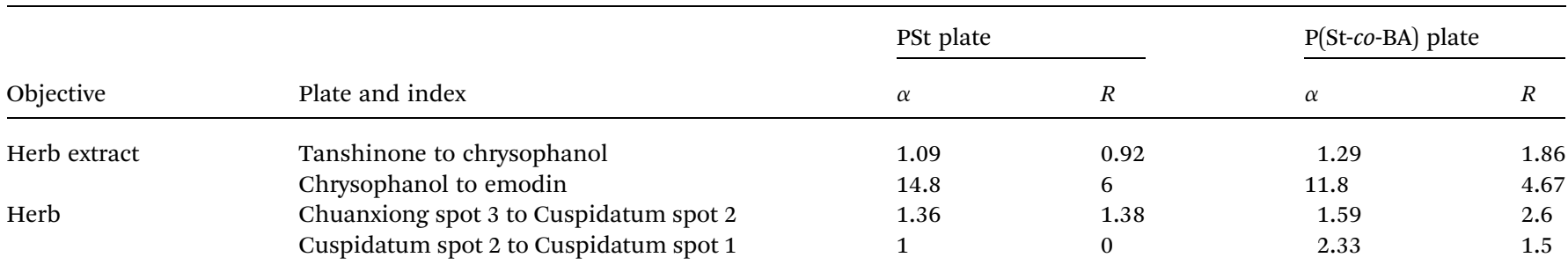

which endowed $\mathrm{P}(\mathrm{St}-\mathrm{co}-\mathrm{BA})$ plate with better performance for TLC analysis, as discussed below.

\subsection{Permeability of polyHIPE}

Permeability of polyHIPE monolith is an important property when used as separation media. During TLC analysis, the mobile phase moves forward on the plate by capillary force and the analytes distribute in the mobile phase and stationary phase. To achieve a partition equilibrium of analytes, a suitable analysis time, i.e. suitable speed of mobile phase is definitely important. Here, we compared the speed of frontier of two typical mobile phases on different plate. As shown in Fig. 5, the speed of frontier on PS10BA20 is much lower than other plates, both in cyclohexane/chloroform $(3: 1)$ and $n$-hexane/ethyl acetate $(9: 1)$ system. Because the analytes can reach partition equilibrium on $\mathrm{P}(\mathrm{St}-c o-\mathrm{BA})$ plate, better separation is achieved on this plate, as discussed below.

\subsection{TLC identification of bioactive compounds in traditional Chinese medicines}

To evaluate the feasibility of TLC by polyHIPE plate, both herb extracts and herbs were chosen as model analytes. Fig. 6 show the typical chromatogram of three coloured herb extracts on $\mathrm{P}(\mathrm{St}-c o-\mathrm{BA})$ plate. Emodin is almost residue at the origin, and $R_{\mathrm{f}}$ of chrysophanol and tanshinone are of $0.59(\mathrm{RSD}=4.3 \%, n=5)$ and 0.76 (RSD $=4.7 \%, n=5$ ), respectively. In Fig. 6b, fluorescent spots of Rhizoma Chuanxiong (spot 3) and Polygonum Cuspidatum (spot 1 and 2) chempresent in the chromatogram of mixture of three herbs. The RSD ( $n=5$ ) of $R_{\mathrm{f}}$ are detected to be $3.3 \%$ for spot $1,4.8 \%$ for spot 2 and $4.9 \%$ for spot 3 , respectively. This polyHIPE plate can be used repeatedly after washed with ethanol to remove impurities. An acceptable separation to three moieties for TLC identification was achieved even after reused ten times (Fig. 6c).

To evaluate the performance of TLC analysis, relative retardation factors $(\alpha)$ and resolution $R$ on PSt and $\mathrm{P}(\mathrm{St}-c o-\mathrm{BA})$ plates are calculated according to eqn (1)-(3) and summarized in Table 2 . The relative retardation factor $(\alpha)$ is related to thermodynamics character and reflects the partition behaviour of analyte in mobile phase and stationary phase. A difference of $\alpha$ is the prerequisite of separation by chromatograph. Here, as shown in Table 2, the $\alpha$ value ranges from 1.0 to 14.8, verifying a large difference in partition behaviour of these analytes. This makes the optimization of chromatograph condition to be practicable in order to obtain good separation. The resolution $R$ is an integrated indicator of the separation degree to two analytes. As a commonly practised criterion, $R>1.0$ is necessary for a basic separation and $R>1.5$ for a complete separation. Based on this criterion, $\mathrm{P}(\mathrm{St}-\mathrm{co}-\mathrm{BA})$ plate presents better performance than PSt plate. As sown in Table 2, when practising on PSt plate, $R$ between tanshinone to chrysophanol is only 0.92 and two spots of Cuspidatum present together $(R=0)$. Contrarily, all the spots mentioned above are completely separated with neighbouring spot $(R>1.5)$ when practising the TLC on $\mathrm{P}(\mathrm{St}-\mathrm{co}-\mathrm{BA})$ plate. Two factors, better homogeneity and partition equilibrium, attributed to the better performance of $\mathrm{P}(\mathrm{St}-\mathrm{co}-\mathrm{BA})$ plate.
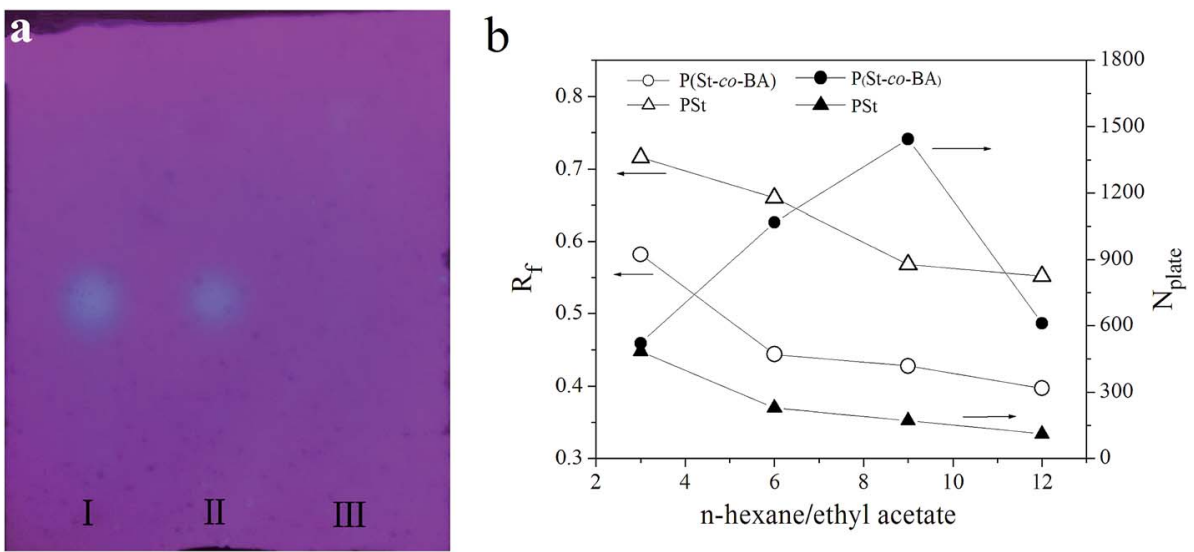

Fig. 7 Identification of Chuanxiong in Bazhen Yumu Wan (a) chromatograms and (b) variation of $R_{\mathrm{f}}$ and $N_{\text {plate }}$ with composition of mobile phase on two plates. I - Chuanxiong herb, II - Bazhen Yumu Wan, III - Chuanxiong negative Bazhen Yumu Wan. 


\subsection{Identification of Chinese traditional medicine by TLC on polyHIPE plate}

Fig. 7a shows the chromatograms for identification of Chuanxiong in Bazhen Yumu Wan, a Chinese traditional medicine made from 9 Chinese herbs. A fluorescence spot $\left(R_{\mathrm{f}} \approx 0.5\right)$ presents in the chromatograms of Chuanxiong and Bazhen Yumu Wan, while it is absent in the chromatogram of Chuanxiong negative Bazhen Yumu Wan. This result verified the feasibility of polyHIPE plate for identification of Chinese traditional medicine. Furthermore, we choose theoretical plate number $\left(N_{\text {plate }}\right)$, to evaluate the performance of plate. $N_{\text {plate }}$ is a core parameter for plate itself and reflects the band broadening during the develop of TLC. Fig. $3 \mathrm{~b}$ shows the $R_{\mathrm{f}}$ and $N_{\text {plate }}$ of fluorescence spot of Chuanxiong under a wide range of mobile phase compositions on PSt and $\mathrm{P}(\mathrm{St}-\mathrm{co}-\mathrm{BA})$ plates. The $R_{\mathrm{f}}$ increase as the ratio of ethyl acetate increase and the $R_{\mathrm{f}}$ on P(St$c o$-BA) plates is larger than $R_{\mathrm{f}}$ on PSt plates under a fixed mobile phase. These verified that both the stationary phase and mobile phase affect the separation, i.e. the analytes interact with both mobile phase and stationary phase. This is the basic principle of chromatographic separation. According to the $N_{\text {plate }}$, best performance was achieved under $n$-hexane/ethyl acetate ratio of $9: 1$ on $\mathrm{P}(\mathrm{St}-\mathrm{co}-\mathrm{BA})$ plate.

\section{Conclusions}

In summary, polyHIPE as stationary phase of TLC was demonstrated for the first time. By polymerized the monomer in the continuous phase of HIPE, open-cellar porous polyHIPE monolithic plate with primary cavities and interconnecting windows was formed as a replica of HIPE in term of shape and internal structure. The prepared monolithic plate was applied as stationary phase of TLC for the identification of herb extracts, herbs and medicines. Some chromatographic parameters such as relative retardation factor, theoretical plate number and resolution were also evaluated and the performance of PSt and $\mathrm{P}(\mathrm{St}-\mathrm{co}$-BA $)$ plates were compared. The introduction of BA in styrenics-based polyHIPE facilitates the preparation and improves the performance for TLC separation. In a word, the developed polyHIPE monolith is a good candidate as stationary phase of TLC with good performance and reusability.

\section{Acknowledgements}

The supports from the graduate starting seed fund of Northwestern Polytechnical University (2017, by Yudong Guan) and the National key research and development program of China (No. 2016YFC0301302) are highly appreciated.

\section{References}

1 P. Bernard-Savary and C. F. Poole, Instrument platforms for thin-layer chromatography, J. Chromatogr. A, 2015, 1421, 184-202.

2 X. Zhong, L. Qiao, B. Liu and H. H. Girault, Ambient in situ analysis and imaging of both hydrophilic and hydrophobic thin layer chromatography plates by electrostatic spray ionization mass spectrometry, $R S C A d v ., 2015$, 5, 7539575402.

3 S. Joseph and F. Bernard, Thin layer chromatographyForeword, J. Liq. Chromatogr. Relat. Technol., 2010, 33, 875879.

4 O. A. Goroshko, O. A. Checha, V. P. Pakhomov, Y. V. Ruvinov and T. D. Ismagilov, Sorbents for thin-layer chromatography in pharmaceutical analysis, Pharm. Chem. J., 2010, 44, 521524.

5 I. I. Hewala, M. M. Bedair and S. M. Shousha, New concept for HPTLC peak purity assessment and identification of drugs in multi-component mixtures, Talanta, 2012, 88, 623-630.

6 Y. Tunç, C. Gölgelioğlu, N. Hasirci, K. Ulubayram and A. Tuncel, Acrylic-based high internal phase emulsion polymeric monolith for capillary electrochromatography, $J$. Chromatogr. A, 2010, 1217, 1654-1659.

7 Y. Tunc, C. Gölgelioğlu, A. Tuncel and K. Ulubayram, Polystyrene-based high internal phase emulsion polymer monolithic stationary phase for capillary electrochromatography, Sep. Sci. Technol., 2012, 47, 24442449.

8 B. Xu, S. Cheng, X. Wang, D. Wang and L. Xu, Novel polystyrene/antibody nanoparticle-coated capillary for immunoaffinity in-tube solid-phase microextraction, Anal. Bioanal. Chem., 2015, 407, 2771-2775.

9 L. Qin, H. Wang and S. Liu, Hollow silica microspheres as the stationary phase for thin layer chromatographic separation of a model mixture, Arabian J. Chem., 2014, DOI: 10.1016/j.arabjc.2014.02.018.

10 M. Amoli-Diva and K. Pourghazi, Gold nanoparticles grafted modified silica gel as a new stationary phase for separation and determination of steroid hormones by thin layer chromatography, J. Food Drug Anal., 2015, 23, 279-286.

11 X. D. Liu, T. Kubo, H. Y. Diao and J. Benjamas, DNA/ polyvinyl alcohol interpenetrating polymer network as stationary phase for thin layer chromatography, Anal. Biochem., 2009, 393, 67-72.

12 H. Gu, D. Yin, J. Ren, B. Zhang and Q. Zhang, Preparation of quaternary amine monolithic column for strong anionexchange chromatography and its application to the separation of Enterovirus 71, J. Chromatogr. B: Anal. Technol. Biomed. Life Sci., 2016, 1033-1034, 399-405.

$13 \mathrm{~J}$. Urban and P. Jandera, Recent advances in the design of organic polymer monoliths for reversed-phase and hydrophilic interaction chromatography separations of small molecules, Anal. Bioanal. Chem., 2013, 405, 2123-2131.

14 R. Bakry, G. K. Bonn, D. Mair and F. Svec, Monolithic porous polymer layer for the separation of peptides and proteins using thin-layer chromatography coupled with MALDITOF-MS, Anal. Chem., 2007, 79, 486-493.

15 Y. Han, P. Levkin, I. Abarientos, H. Liu, F. Svec and J. M. J. Fréchet, Monolithic superhydrophobic polymer layer with photopatterned virtual channel for the separation of peptides using two-dimensional Thin layer 
chromatography-desorption electrospray ionization mass spectrometry, Anal. Chem., 2010, 82, 2520-2528.

16 I. Urbanova and F. Svec, Monolithic polymer layer with gradient of hydrophobicity for separation of peptides using two-dimensional thin layer chromatography and MALDITOF-MS detection, J. Sep. Sci., 2011, 34, 2345-2351.

17 Y. Q. Lv, Z. X. Lin, T. W. Tan and F. Svec, Preparation of porous styrenics-based monolithic layers for thin layer chromatography coupled with matrix-assisted laserdesorption/ionization time-of-flight mass spectrometric detection, J. Chromatogr. A, 2013, 1316, 154-159.

18 K. Wang, Q. C. Chen, Y. Lin, S. Yu and H. Y. Lin, Separation of catechins and O-methylated (-)-epigallocatechin gallate using polyamide thin-layer chromatography, J. Chromatogr. B: Anal. Technol. Biomed. Life Sci., 2016, 1017-1018, 221-225.

19 Y. Zhang, J. Pan, Y. Chen, W. Shi, Y. Yan and L. Yu, HIPEs template: towards the synthesis of polymeric catalysts with adjustable porous structure, acid-base strength and wettability for biomass energy conversation, Chem. Eng. J., 2016, 283, 956-970.

20 H. K. He, W. Li, M. Lamson, M. J. Zhong, D. Konkolewicz and K. Matyjaszewski, Porous polymers prepared via high internal phase emulsion polymerization for reversible $\mathrm{CO}_{2}$ capture, Polymer, 2014, 55, 385-394.

21 M. G. Perez-Garcia, A. Carranza, J. E. Puig, J. A. Pojman and F. del Monte, Porous monoliths synthesized via polymerization of styrene and divinyl benzene in nonaqueous deep-eutectic solvent-based HIPEs, RSC Adv., 2015, 5, 23255-23260.

22 M. S. Silverstein, PolyHIPEs: recent advances in emulsiontemplated porous polymers, Prog. Polym. Sci., 2014, 39, 199-234.

23 M. R. Moghbeli, A. Khajeh and M. Alikhani, Nanosilica reinforced ion-exchange polyHIPE type membrane for removal of nickel ions: preparation, characterization and adsorption studies, Chem. Eng. J., 2017, 309, 552-562.

24 Y. Zhang, Y. Shen, Y. Chen, Y. Yan, J. Pan, Q. Xiong, W. Shi and L. Yu, Hierarchically carbonaceous catalyst with Brønsted-Lewis acid sites prepared through Pickering HIPEs templating for biomass energy conversation, Chem. Eng. J., 2016, 294, 222-235.

25 J. Yang, G. Yang, H. Liu, L. Bai and Q. Zhang, Novel porous monolithic column using poly(high internal phase emulsion) methacrylate as materials for immunoglobulin separation performance on HPLC, Chin. J. Chem., 2010, 28, 229-234.

26 D. Yin, Y. Guan, B. Li and B. Zhang, Antagonistic effect of particles and surfactant on pore structure of macroporous materials based on high internal phase emulsion, Colloids Surf., A, 2016, 506, 550-556.

27 N. Zhang, S. Zhong, X. Zhou, W. Jiang, T. Wang and J. Fu, Superhydrophobic $\mathrm{P}(\mathrm{St}-\mathrm{DVB})$ foam prepared by the high internal phase emulsion technique for oil spill recovery, Chem. Eng. J., 2016, 298, 117-124.

28 A. Menner, M. Salgueiro, M. S. P. Shaffer and A. Bismarck, Nanocomposite foams obtained by polymerization of high internal phase emulsions, J. Polym. Sci., Part A: Polym. Chem., 2008, 46, 5708-5714.

29 D. Yin, B. Q. Li, J. J. Liu and Q. Y. Zhang, Structural diversity of multi-hollow microspheres via multiple Pickering emulsion co-stabilized by surfactant, Colloid Polym. Sci., 2015, 293, 341-347.

30 P. Krajnc, N. Leber, D. Štefanec, S. Kontrec and A. Podgornik, Preparation and characterisation of poly(high internal phase emulsion) methacrylate monoliths and their application as separation media, J. Chromatogr. A, 2005, 1065, 69-73. 